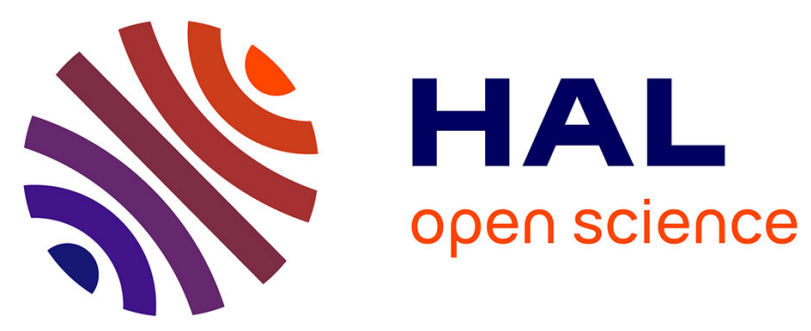

\title{
A Differential Permeability 3-D Formulation for Anisotropic Vector Hysteresis Analysis
}

Joao Pedro A. Bastos, Nelson Sadowski, J. V. Leite, Nelson Jhoe Batistela, Kleyton Hoffmann, Gérard Meunier, Olivier Chadebec

\section{- To cite this version:}

Joao Pedro A. Bastos, Nelson Sadowski, J. V. Leite, Nelson Jhoe Batistela, Kleyton Hoffmann, et al.. A Differential Permeability 3-D Formulation for Anisotropic Vector Hysteresis Analysis. IEEE Transactions on Magnetics, 2014, 50 (2), pp.réf article: 7008304. 10.1109/TMAG.2013.2282697. hal-00957434

\section{HAL Id: hal-00957434 \\ https://hal.science/hal-00957434}

Submitted on 26 Jan 2021

HAL is a multi-disciplinary open access archive for the deposit and dissemination of scientific research documents, whether they are published or not. The documents may come from teaching and research institutions in France or abroad, or from public or private research centers.
L'archive ouverte pluridisciplinaire HAL, est destinée au dépôt et à la diffusion de documents scientifiques de niveau recherche, publiés ou non, émanant des établissements d'enseignement et de recherche français ou étrangers, des laboratoires publics ou privés. 


\title{
A Differential Permeability 3-D Formulation for Anisotropic Vector Hysteresis Analysis
}

\author{
Joao Pedro A. Bastos ${ }^{1}$, Nelson Sadowski ${ }^{1}$, Jean Vianei Leite ${ }^{1}$, Nelson Jhoe Batistela ${ }^{1}$, \\ Kleyton Hoffmann ${ }^{1}$, Gérard Meunier ${ }^{2}$, and Olivier Chadebec ${ }^{2}$ \\ ${ }^{1}$ GRUCAD/EEL/CTC, Universidade Federal de Santa Catarina, Florianópolis 88040-900, Brazil \\ ${ }^{2}$ G2Elab, UMR CNRS 5269, Grenoble INP, Universit Joseph Fourier, Saint Martin d'Hères 38400, France
}

In the previous work, we introduced a formulation joining two concepts: 1) the source-field technique and 2) the differential permeability method. It was shown that the resulting procedure produces accurate results for non-linear cases and it was favorably compared with the more classical approach considering the actual permeability. Here, we extend this formulation for vector hysteresis cases that are modeled by the Jiles-Atherton approach. It considers the magnetic anisotropy of the ferromagnetic sheets.

Index Terms-Finite element methods (FEMs), magnetic anisotropy, vector hysteresis.

\section{INTRODUCTION}

$\mathbf{M}$ ODELING electrical devices possessing ferromagnetic sheets under variable magnetic fields may require, in certain cases, a high degree of accuracy. If the magnetic circuit does not have air gaps (as transformer yokes), hysteresis plays a relevant role on the device magnetic behavior. The problem becomes more complex if one wishes to consider the electrical sheets anisotropy [2] and the electrical circuit coupling.

In [1], we introduced a formulation based on differential permeability coupled to the source-field method for solving non-linear problems. The source-field method has been previously developed and presented in several papers and it is also commonly called T- $\Omega$ method [3]-[6]. When proposing [1], we pointed out that such an approach would allow solving hysteretic cases, which is now considered in this paper. The hysteresis is modeled by the Jiles-Atherton (JA) approach [7], [8]. The work developed in [9] differs from ours since it employs the magnetic vector potential and the inverse JA model. An interesting paper related to ours is proposed in [10]; it differs from this paper using formulations often applied for losses evaluation and the vector hysteresis was not considered. Although the majority of the concepts used in this paper (anisotropy, differential permeability tensor, JA technique, electric circuit coupling, and source-field method) are well known, assembling them all in a 3-D FE program is the original contribution of this paper. To demonstrate the effectiveness of the proposed method, the TEAM Workshop Problem 32 is solved.

\section{FORMULATION}

With the differential permeability method, we have, for nonlinear materials

$$
\Delta \mathbf{B}=\left\|\mu_{d}\right\| \Delta \mathbf{H}
$$

where $\left\|\mu_{d}\right\|$ is the differential permeability tensor. $\mathbf{H}$ and $\mathbf{B}$ are magnetic field and induction, respectively. For the proposed vector hysteresis modeling, we utilize a time step procedure considering the differential permeability as a tensor.
As the time evolutes from $t-\Delta t$ to $t$, the corresponding time steps are $i-1$ and $i$. The variations of $\mathbf{H}$ and $\mathbf{B}$ are

$$
\Delta \mathbf{B}=\mathbf{B}^{i}-\mathbf{B}^{i-1} \text { and } \Delta \mathbf{H}=\mathbf{H}^{i}-\mathbf{H}^{i-1} .
$$

With the source-field method [1], [3], we have

$$
\mathbf{H}^{i}=\mathbf{H}_{s}^{i}-\operatorname{grad} \Omega^{i}
$$

where $\Omega$ is the magnetic scalar potential and $\mathbf{H}_{s}$ is the source field associated to the imposed current density as $\operatorname{rot} \mathbf{H}_{s}=\mathbf{J}$ [1], [3]. $\mathbf{H}^{i-1}$ and $\mathbf{B}^{i-1}$ are known from the previous time step.

Considering $\mathbf{B}^{i}=\Delta \mathbf{B}+\mathbf{B}^{i-1}$, (1), (2) and (3), we have

$$
\mathbf{B}^{i}=\mathbf{B}^{i-1}+\left\|\mu_{d}\right\|\left(\mathbf{H}_{s}^{i}-\operatorname{grad} \Omega^{i}-\mathbf{H}^{i-1}\right) .
$$

The main equation on this development is

$$
\begin{gathered}
\operatorname{div} \mathbf{B}^{i}=0 \\
\text { or } \quad \operatorname{div}\left[\mathbf{B}^{i-1}+\left\|\mu_{d}\right\|\left(\mathbf{H}_{s}^{i}-\operatorname{grad} \Omega^{i}-\mathbf{H}^{i-1}\right)\right]=0 .
\end{gathered}
$$

Applying the Galerkin method on (5), we have

$$
\int_{V} N \operatorname{div} \mathbf{B}^{i} d v=0
$$

where $N$ is the nodal shape function of a tetrahedron element and $V$ is the domain volume. It can be written as

$$
\int_{V} N \operatorname{div} \mathbf{B}^{i} d v=\oint_{s(v)} N \mathbf{B}^{i} \cdot d \mathbf{s}-\int_{V} \operatorname{grad} N \cdot \mathbf{B}^{i} d v=0 .
$$

The first term on the right-hand side is related to the classical boundary conditions for scalar potential [1]. The second one must be evaluated for the numerical implementation as

$$
-\int_{V} \operatorname{grad} N \cdot \mathbf{B}^{i} d v=0
$$

or, using (4)

$-\int_{V} \operatorname{grad} N \cdot\left[\mathbf{B}^{i-1}+\left\|\mu_{d}\right\|\left(\mathbf{H}_{s}^{i}-\operatorname{grad} \Omega^{i}\right)-\left\|\mu_{d}\right\| \mathbf{H}^{i-1}\right] d v=0$.

Let us remember that the vectors $\mathbf{N}$ and $\mathbf{K}$, already presented in [1], are related by

$$
\mathbf{H}_{s}=\mathbf{K} I_{0}
$$


where $I_{0}$ is the current flowing in a coil conductor; $\mathbf{K}$ is the corresponding source field when this current is equal $1 \mathrm{~A}$. Also, we will use the vector $\mathbf{N}$ expressed by

$$
\operatorname{curl} \mathbf{H}_{s}=\mathbf{N} I_{0} .
$$

With (10) in the equation above, we have curl $\mathbf{K} I_{0}=\mathbf{N} I_{0}$ and

$$
\operatorname{curl} \mathbf{K}=\mathbf{N}
$$

where $\mathbf{K}$ corresponds to the magnetic field created by an unitary current in a coil wire. $\mathbf{N}$ and $\mathbf{K}$ are necessary to accomplish the coupling between the electric circuit and the magnetic device. Now, let us consider the electric circuit equation

$$
V_{m}=R_{m} i_{m}+\frac{d \Phi_{m}}{d t}
$$

where $V_{m}, R_{m}, i_{m}$, and $\Phi_{m}$ are, respectively, the voltage, the resistance, the established current, and the magnetic flux linkage in a generic electric circuit $m$. From [1], it can be shown that the magnetic flux is given by

$$
\Phi^{i}=\int_{V_{b}} \mathbf{B}^{i} \cdot \mathbf{K} d v
$$

For the time step $i$, we have

$$
V_{m}{ }^{i}=R_{m} i_{m}{ }^{i}+\frac{d}{d t} \int_{V_{b}} \mathbf{B}^{i} \cdot \mathbf{K} d v .
$$

Here, we apply the derivative time discretization as

$$
\begin{aligned}
& \Delta t V_{m}{ }^{i}=\Delta t R_{m} i_{m}{ }^{i}+\int_{V_{b}} \Delta \mathbf{B}^{i} \cdot \mathbf{K} d v \\
& \Delta t V_{m}{ }^{i}=\Delta t R_{m} i_{m}{ }^{i}+\int_{V_{b}}\left(\mathbf{B}^{i}-\mathbf{B}^{i-1}\right) \cdot \mathbf{K} d v .
\end{aligned}
$$

Using (4) we obtain

$$
\begin{aligned}
& \Delta t V_{m}{ }^{i}=\Delta t R_{m} i_{m}{ }^{i} \\
& +\int_{V_{b}}\left(\mathbf{B}^{i-1}+\left\|\mu_{d}\right\|\left(\mathbf{H}_{s}{ }^{i}-\operatorname{grad} \Omega^{i}-\mathbf{H}^{i-1}\right)-\mathbf{B}^{i-1}\right) \cdot \mathbf{K} d v \\
& \Delta t V_{m}{ }^{i}=\Delta t R_{m} i_{m}{ }^{i}+\int_{V_{b}}\left(\left\|\mu_{d}\right\|\left(\mathbf{H}_{s}{ }^{i}-\operatorname{grad} \Omega^{i}-\mathbf{H}^{i-1}\right)\right) \cdot \mathbf{K} d v .
\end{aligned}
$$

Using a simplified notation $G=\operatorname{grad} N$ and also considering that the numerical integration is performed, (16) becomes

$\Delta t V_{m}^{i}=\Delta t R_{m} i_{m}{ }^{i}$

$+\left(K^{T}\left\|\mu_{d}\right\| K i_{m}{ }^{i}-K^{T}\left\|\mu_{d}\right\| G \Omega^{i}-K^{T}\left\|\mu_{d}\right\| H^{i-1}\right)$.

Using (10), equation (9) is

$\int_{V}-\operatorname{grad} N \cdot\left[\mathbf{B}^{i-1}+\left\|\mu_{d}\right\|\left(\mathbf{K} i_{m}{ }^{i}-\operatorname{grad} \Omega^{i}\right)-\left\|\mu_{d}\right\| \mathbf{H}^{i-1}\right] d v=0$ and, with the adopted notations

$$
\begin{aligned}
& -G^{T} B^{i-1}-G^{T}\left\|\mu_{d}\right\| K i_{m}{ }^{i}+G^{T}\left\|\mu_{d}\right\| G \Omega^{i} \\
& +G^{T}\left\|\mu_{d}\right\| H^{i-1}=0 .
\end{aligned}
$$

Arranging (17) and (18) in matrix form, we have

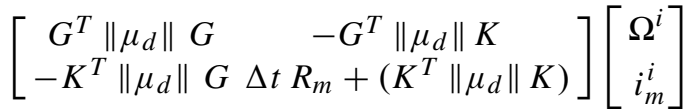

$$
\begin{aligned}
& =\left[\begin{array}{c}
G^{T} B^{i-1}-G^{T}\left\|\mu_{d}\right\| H^{i-1} \\
\Delta t V_{m}^{i}+K^{T}\left\|\mu_{d}\right\| H^{i-1}
\end{array}\right] .
\end{aligned}
$$

Since we are dealing with several terms and matrix products in (19), it seems worthy to provide an implementation information related to array dimensions of terms (19). First, for a tetrahedron elements, $G=\operatorname{grad} N$ is a $(3,4)$ matrix. $\mathbf{K}$ is a vector depending on the number of circuits. Let us suppose that there are two circuits and then $K$ is a $(3,2)$ matrix. We observe that $\mathbf{K}$ is related to $\mathbf{H}$, which is calculated with tetrahedron edge elements [1]. Therefore, $K$ is obtained by the product of the edge shape functions $\mathbf{w}(3,6)$ by the circulation of the source field (as defined in (11)) along the six edges. There are two sets of such circulations since there are two circuits in this example. $R$ is related to the circuit resistances. Supposing also two circuits, $R$ is $(2,1)$ matrix. Now, let us consider the terms of (19). The term dimensions are:

1) for $G^{T}\left\|\mu_{d}\right\| G:(4,3)$. (3, 3) . (3, 4) $\rightarrow(4,4)$;

2) for $G^{T}\left\|\mu_{d}\right\| K:(4,3) .(3,3) .(3,2) \rightarrow(4,2)$;

3) for $G^{T} B^{i-1}:(4,3)$. $(3,1) \rightarrow(4,1)$;

4) for $G^{T}\left\|\mu_{d}\right\| H^{i-1}:(4,3) .(3,3) .(3,1) \rightarrow(4,1)$;

5) for $K^{T}\left\|\mu_{d}\right\| K:(2,3)$. (3, 3) . (3, 2) $\rightarrow(2,2)$;

6) for $K^{T}\left\|\mu_{d}\right\| H^{i-1}:(2,3)$. $(3,3) .(3,1) \rightarrow(2,1)$.

As remark, the first four matrices above are assembled in the first line of (19), since they are related to the tetrahedra. The last two ones are assembled in the second line of (19) related to the two electric circuits.

\section{Permeability Tensor By the JA Model}

If the magnetic flux rotates in an electrical sheet, the vector relationship between field and induction plays an important role in the device modeling. If one wants to consider the saturation effect as well as the hysteresis losses with the rotating flux, it is imperative to employ a vector hysteresis model.

Recently, several models have been proposed. Bergqvist [11], based on the scalar JA hysteresis model [12], proposed a vector model whose independent variable is the vector magnetic field. Leite et al. [7] proposed some modifications in the vectorized model in order to improve its numerical performance. In this last work, the model main equations are as follows:

$$
\begin{aligned}
d \mathbf{M} & =\left\{\mathbf{1}-F_{\chi} \cdot \overleftrightarrow{\alpha}-\overleftrightarrow{c} \cdot \overleftrightarrow{\xi} \cdot \overleftrightarrow{\alpha}\right\}^{-1} \cdot\left\{F_{\chi}+\overleftrightarrow{c} \cdot \overleftrightarrow{\xi}\right\} d \mathbf{H} \\
F_{\chi} & =\vec{\chi}_{f}\left|\vec{\chi}_{f}\right|^{-1} \cdot \vec{\chi}_{f} \\
\overleftrightarrow{\chi}_{f} & =\stackrel{\leftrightarrow}{k}^{-1} \cdot\left(\mathbf{M}_{a n}-\mathbf{M}\right) \\
\mathbf{M}_{a n} & =\mathbf{M}_{a n}\left(\left|\mathbf{H}_{e}\right|\right) \frac{\mathbf{H}_{e}}{\left|\mathbf{H}_{e}\right|} .
\end{aligned}
$$

where $\mathbf{M}$ is the total magnetization, $\mathbf{H}$ is the magnetic field vector, $\mathbf{1}$ is the identity matrix, $\vec{\chi}_{f}$ is an auxiliary vector, $\mathbf{M}_{a n}$ is the anhystereric magnetization given by the Langevin equation vector form, $\mathbf{H}_{e}$ is the effective magnetic field given by $\mathbf{H}_{e}=\mathbf{H}+\stackrel{\leftrightarrow}{\alpha} \cdot \mathbf{M}$, and $\stackrel{\leftrightarrow}{\xi}$ is a matrix containing the derivatives of $\mathbf{M}_{a n}$ with respect to $\mathbf{H}_{e}$ [7]. The tensors $\overleftrightarrow{\alpha}, \stackrel{\leftrightarrow}{c}$, and $\overleftrightarrow{k}$ are related to material parameters. Two additional tensors not shown in equations above $\left(\overleftrightarrow{M}_{S}\right.$ and $\left.\stackrel{\leftrightarrow}{a}\right)$ with parameters related to the Langevin function are also necessary [7].

$\left\|\mu_{d}\right\|$ can be obtained from (20) with the aid of the constitutive law $d \mathbf{B}=\mu_{0}(d \mathbf{H}+d \mathbf{M})$. 


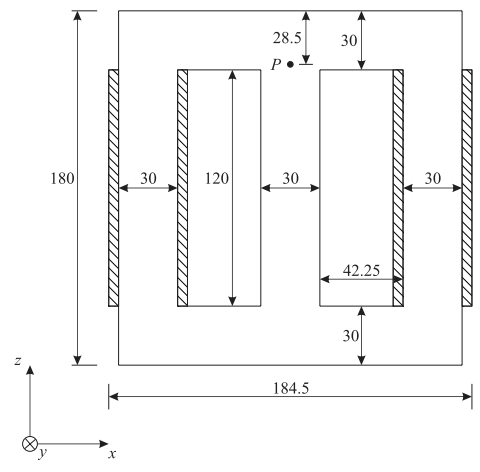

Fig. 1. Device structure (dimensions in $\mathrm{mm}$ ).

Using (20), one can obtain the differential permeability tensor by the equation

$d \mathbf{B}=\mu_{0}\left(\mathbf{1}+\left\{\mathbf{1}-F_{\chi} \cdot \overleftrightarrow{\alpha}-\overleftrightarrow{c} \cdot \overleftrightarrow{\xi} \cdot \overleftrightarrow{\alpha}\right\}^{-1} \cdot\left\{F_{\chi}+\overleftrightarrow{c} \cdot \overleftrightarrow{\xi}\right\}\right) d \mathbf{H}$.

Some advantages of this model are its relative mathematical simplicity, easy numerical implementation, small parameters set, and numerical robustness. Furthermore, the model can represent anisotropy and reduces itself to a scalar model if the magnetic flux is unidirectional. More details about the model characteristics and implementation can be found in [7] and [11].

\section{EXAMPLE}

As an example, we present the case proposed by the TEAM Problem 32 [13]. In this case, it is necessary to utilize a vector hysteresis modeling since rotating fields are present in some regions of the structure. In Fig. 1, there is a threelimbed ferromagnetic core. The core has five Fe-Si $0.48 \mathrm{~mm}$ thick sheets. Therefore, the depth of the device $(2.4 \mathrm{~mm}$ in $O y$-direction) is much smaller than $O z x$ dimensions. It creates an additional difficulty, since the mesh may have a poor quality factor unless it is very fine (which would affect the computational time). This case could be calculated as a 2-D case, although our calculations were performed with the 3-D formulation here proposed. This problem has four different variations but we are interested on the CASE3 (as denoted in [13]), which is appropriated for checking vector hysteresis modeling. Two windings having 90 turns each are placed on the external limbs and the voltage sources are shifted $90^{\circ}$. Each coil has a total resistance (external resistances are connected to the windings) of $11.42 \Omega$. The peak voltage is, for both, $14.5 \mathrm{~V}$ and the frequency is $10 \mathrm{~Hz}$. The full description of the problem can be found in [13].

We provided the experimental hysteresis data in the vertical and horizontal directions. For their simulation, they used Preisach modeling [14]. Using an optimizing procedure, we found the JA parameters presented in Table I. The corresponding $B(H)$ curve for the rolling (vertical) direction is shown in Fig. 2. There is a very good agreement between experimental and simulated hysteresis loops. The same agreement is also found for the transverse direction. The magnetic field in $O y$-direction is negligible and, for this direction, we use parameters identical to $O x$ ones.

Because many calculation attempts were necessary and each simulation needed several hours, we chose a relatively
TABLE I

PARAMETERS OF THE JA MODEL

\begin{tabular}{c||ccc}
\hline & $O x$ Direction & $O y$ Direction & $O z$ Direction \\
\hline \hline$M_{s}$ & $1.31 \times 10^{6}[\mathrm{~A} / \mathrm{m}]$ & $1.31 \times 10^{6}[\mathrm{~A} / \mathrm{m}]$ & $1.33 \times 10^{6}[\mathrm{~A} / \mathrm{m}]$ \\
$k$ & $374.975[\mathrm{~A} / \mathrm{m}]$ & $374.975[\mathrm{~A} / \mathrm{m}]$ & $232.652[\mathrm{~A} / \mathrm{m}]$ \\
$c$ & $736 \times 10^{-3}$ & $736 \times 10^{-3}$ & $652 \times 10^{-3}$ \\
$a$ & $233.78[\mathrm{~A} / \mathrm{m}]$ & $233.78[\mathrm{~A} / \mathrm{m}]$ & $172.856[\mathrm{~A} / \mathrm{m}]$ \\
$\alpha$ & $562 \times 10^{-6}$ & $562 \times 10^{-6}$ & $417 \times 10^{-6}$ \\
\hline
\end{tabular}

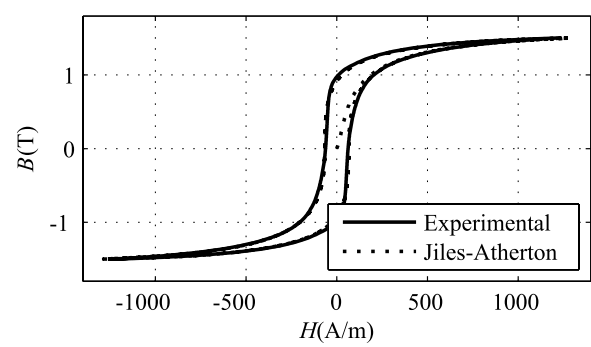

Fig. 2. Experimental and calculated $B(H)$ curves for vertical direction.

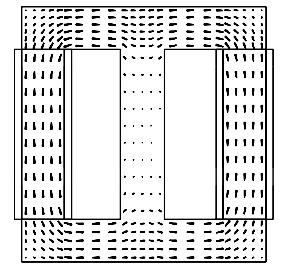

(a)

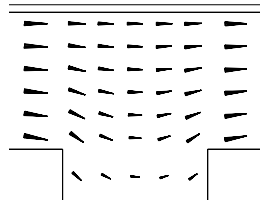

(b)

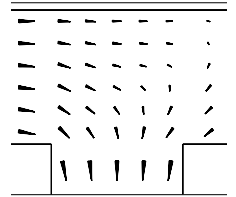

(c)

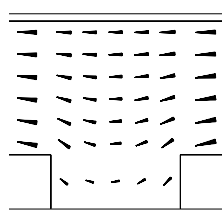

(d)

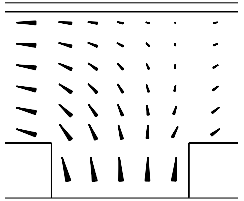

(e)
Fig. 3. Inductions in the structure. (a) $t=0.018 \mathrm{~s}$. (b) $t=0.018 \mathrm{~s}$. (c) $t=0.036 \mathrm{~s}$. (d) $t=0.062 \mathrm{~s}$. (e) $t=0.086 \mathrm{~s}$.

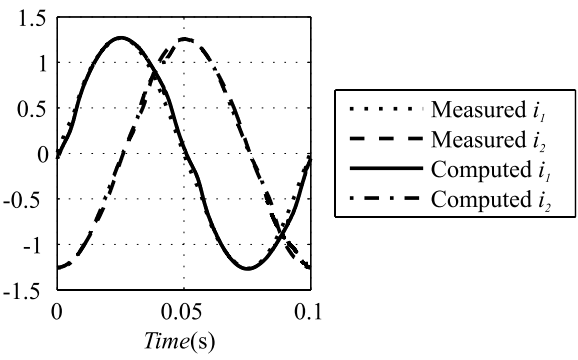

Fig. 4. Current at the windings.

coarse mesh with 9450 tetrahedron elements and 2288 nodes. As a graphical result, the following Fig. 3 shows the magnetic induction at four different time steps. Fig. 3(a) shows the whole structure. The other figures present the magnetic induction vector around the point $P$ shown in Fig. 1. The rotational behavior of fields is easily observed.

Fig. 4 shows the experimental and measured currents on the two windings and the results present excellent agreement.

For local quantities, we present the magnetic induction on the point $P$ in Fig. 1. In Fig. 5(a), the $O z$-direction 


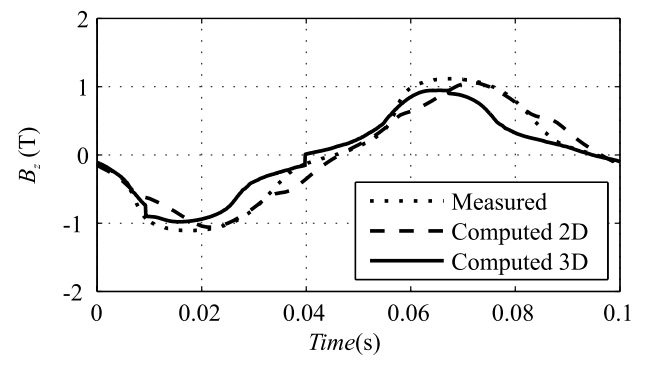

(a)

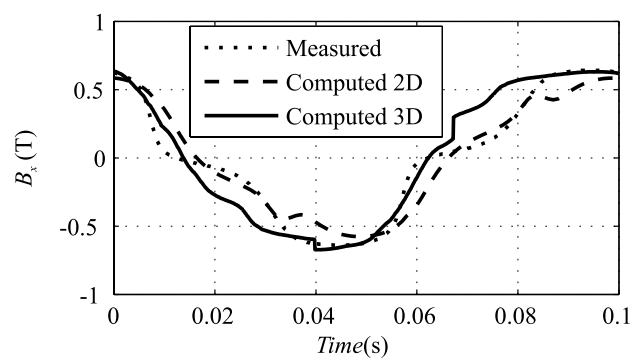

(b)

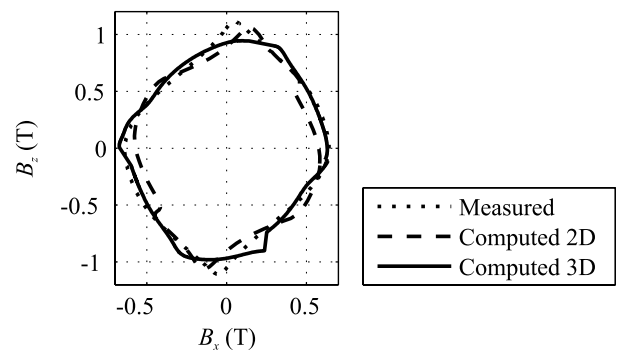

(c)

Fig. 5. Inductions at the point $P$. (a) $O_{z}$ magnetic induction at point $P$. (b) $O_{x}$ magnetic induction at point $P$. (c) B-loci at point $P$.

induction values from the experimental bench and from the simulation are presented. In the Fig. 5(b), we have similar results for the $O x$-direction. And the B-loci obtained from the previous Fig. 5(a) and (b) is shown in the Fig. 5(c). We also included results obtained from our 2-D FE system intended to solve vector hysteresis cases [15]. There are some differences among these results. When performing comparisons, it must be noticed that the measured inductions are obtained through pick-up coils. These coils are $7.3 \mathrm{~mm}$ large in $O x$ - and $O z$-directions, which represents a relatively large dimension. Therefore, measurements are obtained from magnetic fluxes, which are integral quantities and certainly smooth the measured values.

When considering convergence, two aspects must be seen:

1) for solving the linear system, the ICCG method is used; it normally converges well even though, in few time steps, the convergence is not reached; it is certainly related to the poor quality mesh, as already commented;

2) for the non-linearity related to $B(H)$ material characteristics, at some time steps, the convergence is critical.

To mitigate such difficulties, it is possible to apply some numerical techniques as variable time steps and under relaxation. The choice of the time step and the mesh coarseness play a considerable role on the convergence and the result accuracy. For one cycle 1200 time steps were applied.
Solutions for this TEAM problem have been seldom reported. In [16] results are shown. In this paper, we use current-driven windings (no coupling with the voltage sources) and the vector hysteresis phenomenon is considered only on parts of the yoke. In our simulation, the vector JA anisotropic model is used on the whole structure and the device is voltage fed. The numerical simulation is then simplified but it must be mentioned that, as in our simulation, their results present good accuracy. Considering the above analysis and in spite of some result differences, we estimate that our formulation is consistent.

\section{CONCLUSION}

In this paper, we presented a formulation for solving vector hysteresis cases considering the coupling with electrical circuits. The inherent convergence difficulties were also discussed and the strategies to overcome them were applied with efficiency. The TEAM Workshop 32 was used for testing the proposed method and the results show a good agreement between measured and computed results.

\section{REFERENCES}

[1] J. P. A. Bastos and N. Sadowski, "A new formulation using differential permeability based on the source-field method," IEEE Trans. Magn., vol. 46, no. 8, pp. 3369-3372, Aug. 2010.

[2] J. P. A. Bastos and G. Quichaud, "3D modelling of a nonlinear anisotropic lamination," IEEE Trans. Magn., vol. 21, no. 6, pp. 2366-2369, Nov. 1985.

[3] Y. Le Menach, S. Clenet, and F. Piriou, "Determination and utilization of the source field in 3D magnetostatic problems," IEEE Trans. Magn., vol. 34, no. 5, pp. 2509-2512, Sep. 1998.

[4] O. Biro, K. Preis, G. Vrisk, K. R. Richter, and I. Ticar, "Computation of 3-D magnetostatic fields using a reduced scalar potential," IEEE Trans. Magn., vol. 29, no. 5, pp. 1329-1332, Mar. 1993.

[5] J. Simkin and C. W. Trowbridge, "On the use of the total scalar potential in the numerical solution of field problems in electromagnetics," Int. J. Numer. Math. Eng., vol. 14, no. 2, pp. 423-440, 1979.

[6] C. J. Carpenter, "Comparison of alternative formulations of 3-dimensional magnetic-field and eddy-current problems at power frequencies," Proc. Inst. Electr. Eng., vol. 124, no. 11, pp. 1026-1034, 1997.

[7] J. V. Leite, N. Sadowski, P. Kuo-Peng, N. J. Batistela, J. P. A. Bastos, and A. A. de Espíndola, "Inverse Jiles-Atherton vector hysteresis model," IEEE Trans. Magn., vol. 40, no. 4, pp. 1769-1775, Jul. 2004.

[8] J. V. Leite, A. Benabou, and N. Sadowski, "Transformer inrush currents taking into account vector hysteresis," IEEE Trans. Magn., vol. 46, no. 8, pp. 3237-3240, Aug. 2010

[9] J. V. Leite, A. Benabou, N. Sadowski, S. Clenet, J. P. A. Bastos, and F. Piriou, "Implementation of an anisotropric vector hysteresis model in a 3-D FE code," IEEE Trans. Magn., vol. 44, no. 6, pp. 918-921, Jan. 2008.

[10] D. Lin, P. Zhou, W. N. Fu, Z. Badics, and Z. J. Cendes, "A dynamic core loss model for soft ferromagnetic and power ferrite materials in transient finite element analysis," IEEE Trans. Magn., vol. 40, no. 2, pp. 1318-1321, Mar. 2004.

[11] A. J. Bergqvist, "A simple vector generalization of the Jiles-Atherton model of hysteresis," IEEE Trans. Magn., vol. 32, no. 5, pp. 4213-4215, Sep. 1996.

[12] D. C. Jiles and D. L. Atherton, "Theory of ferromagnetic hysteresis," J. Magn. Mater, vol. 61, nos. 1-2, pp. 48-60, 1986.

[13] O. Bottauscio, M. Chiampi, C. Ragusa, L. Rege, and M. Repetto, (2010). Description of TEAM Problem: 32 A Test Case for Validation of Magnetic Field Analysis with Vector Hysteresis [Online]. Available: http://www.compumag.co.uk/teamindex.html

[14] I. D. Mayergoyz, Mathematical Models of Hysteresis, New York, NY, USA: Springer-Verlag, 1991.

[15] J. V. Leite, A. Benabou, N. Sadowski, and M. V. F. da Luz, "Finite element three-phase transformer modeling taking into account a vector hysteresis model," IEEE Trans. Magn., vol. 45, no. 3, pp. 1716-1719, Mar. 2009.

[16] X. Wang, D. Xie, B. Bai, N. Takahashi, and S. Yang, "3-D FEM analysis in electromagnetic system considering vector hysteresis and anisotropy," IEEE Trans. Magn., vol. 44, no. 6, pp. 890-893, Jun. 2008. 